\title{
Smoking and Risk for Amyotrophic Lateral Sclerosis: Analysis of the EPIC Cohort
}

Valentina Gallo, MD, MSc, ${ }^{1,2} \mathrm{H}$. Bas Bueno-de-Mesquita, MD, MPH, PhD, ${ }^{3}$ Roel Vermeulen, PhD, ${ }^{4,5}$ Peter M. Andersen, MD, DMSc, ${ }^{6}$ Andreas Kyrozis, MD, PhD, ${ }^{7}$ Jakob Linseisen, $\mathrm{PhD},{ }^{8}$ Rudolph Kaaks, PhD, ${ }^{8}$ Naomi E. Allen, DPhil, ${ }^{9}$ Andrew W. Roddam, DPhil, ${ }^{9}$ Hendriek C. Boshuizen, PhD, ${ }^{3}$

Petra H. Peeters, MD, PhD, ${ }^{4}$ Domenico Palli, MD, ${ }^{10}$ Amalia Mattiello, MD, ${ }^{11}$ Sabina Sieri, PhD, ${ }^{12}$

Rosario Tumino, MD, ${ }^{13}$ Juan-Manuel Jiménez-Martín, BSc, ${ }^{14}$ María José Tormo Díaz, MD, ${ }^{15,16}$

Laudina Rodriguez Suarez, MD, MPH, ${ }^{17}$ Antonia Trichopoulou, MD, ${ }^{18}$ Antonio Agudo, MD, MSc, PhD, ${ }^{19}$

Larraitz Arriola, MD, MSc ${ }^{20}$ Aurelio Barricante-Gurrea, MD,${ }^{16,21}$ Sheila Bingham, $\mathrm{PhD},{ }^{22}$

Kay-Tee Khaw, MBBChir, ${ }^{23}$ Jonas Manjer, MD, PhD, ${ }^{24}$ Björn Lindkvist, MD, PhD, ${ }^{25}$ Kim Overvad, PhD, ${ }^{26}$ Flemming W. Bach, MD, ${ }^{27}$ Anne Tjønneland, MD, PhD, ${ }^{28}$ Anja Olsen, MSc, PhD, ${ }^{28}$

Manuela M. Bergmann, PhD, ${ }^{29}$ Heiner Boeing, $\mathrm{PhD},{ }^{29}$ Francoise Clavel-Chapelon, PhD, ${ }^{30}$

Eiliv Lund, MD, PhD, ${ }^{31}$ Göran Hallmans, MD, PhD, ${ }^{32}$ Lefkos Middleton, MD, FRCP, ${ }^{2}$

Paolo Vineis, MD, MPH, FFPH, ${ }^{1}$ and Elio Riboli, MD, MSc, $\mathrm{MPH}^{1}$

\begin{abstract}
Objective: Cigarette smoking has been reported as "probable" risk factor for Amyotrophic Lateral Sclerosis (ALS), a poorly understood disease in terms of aetiology. The extensive longitudinal data of the European Prospective Investigation into Cancer and Nutrition (EPIC) were used to evaluate age-specific mortality rates from ALS and the role of cigarette smoking on the risk of dying from ALS.

Methods: A total of 517,890 healthy subjects were included, resulting in 4,591,325 person-years. ALS cases were ascertained through death certificates. Cox hazard models were built to investigate the role of smoking on the risk of ALS, using packs/years and smoking duration to study dose-response.

Results: A total of 118 subjects died from ALS, resulting in a crude mortality rate of 2.69 per 100,000/year. Current smokers at recruitment had an almost two-fold increased risk of dying from ALS compared to never smokers $(\mathrm{HR}=1.89,95 \%$ C.I. $1.14-3.14)$, while former smokers at the time of enrolment had a $50 \%$ increased risk ( $\mathrm{HR}=1.48,95 \%$ C.I. $0.94-2.32)$. The number of years spent smoking increased the risk of ALS ( $p$ for trend $=0.002$ ). Those who smoked more than 33 years had more than a two-fold increased risk of ALS compared with never smokers (HR $=2.16,95 \%$ C.I. 1.33-3.53). Conversely, the number of years since quitting smoking was associated with a decreased risk of ALS compared with continuing smoking.

Interpretation: These results strongly support the hypothesis of a role of cigarette smoking in aetiology of ALS. We hypothesize that this could occur through lipid peroxidation via formaldehyde exposure.
\end{abstract}

From the ${ }^{1}$ Division of Epidemiology, Public Health and Primary Care, Imperial College London; ${ }^{2}$ Division of Neuroscience and Mental Health, Imperial College London, London, United Kingdom; ${ }^{3}$ National Institute for Public Health and the Environment (RIVM), Bilthoven; ${ }^{4}$ Julius Centre, University Medical Centre Utrecht; ${ }^{5}$ Institute for Risk Assessment Sciences (IRAS) University, Utrecht, The Netherlands; 'Department of Neurology, Umeå University Hospital, Umeå, Sweden; ${ }^{7}$ Department of Neurology, Eginition Hospital, University of Athens Medical School, Athens, Greece; ${ }^{8}$ German Cancer Research Centre, Heidelberg, Germany; ${ }^{9}$ Cancer Epidemiology Unit, University of Oxford, Oxford, United Kingdom; ${ }^{10}$ Molecular and Nutritional Epidemiology Unit, Cancer Research and Prevention Institute (ISPO), Florence; ${ }^{11}$ Department of Clinical and Experimental Medicine, Federico II University, Naples; ${ }^{12}$ Nutritional Epidemiology Unit, National Cancer Institute, Milan; ${ }^{13}$ Ragusa Cancer Registry, Azienda Ospedaliera "Civile MP Arezzo," Ragusa, Italy; ${ }^{14}$ Andalusian School of Public Health, Granada; ${ }^{15}$ Epidemiology Service Murcia Health Council, Murcia, Spain; ${ }^{16}$ Centro de Investigación Biomédica en Red (CIBER) Epidemiología y Salud Pública; ${ }^{17}$ Consejería de Salud y Servicios Sanitarios, Principado de Asturias, Spain; ${ }^{18}$ Department of Hygiene and Epidemiology, University of Athens Medical School, Athens, Greece; ${ }^{19}$ Unit of Nutrition, Environment, and Cancer, Catalan Institute of Oncology, Barcelona; ${ }^{20}$ Public Health Division of Gipuzkoa, Donostia-San Sebastian; ${ }^{21}$ Public Health Institute of Navarra, Pamplona, Spain; ${ }^{22}$ Medical Research Council Dunn Human Nutrition
Unit and ${ }^{23}$ Clinical Gerontology Unit, University of Cambridge, Cambridge, United Kingdom; ${ }^{24}$ Department of Surgery, Malmö University Hospital, Malmö; ${ }^{25}$ Department of Internal Medicine, Sahlgrenska University Hospital, Gothenburg, Sweden; Departments of ${ }^{26}$ Clinical Epidemiology and ${ }^{27}$ Neurology, Aarhus University Hospital, Aalborg; ${ }^{28}$ Institute of Cancer Epidemiology, Danish Cancer Society, Copenhagen, Denmark; ${ }^{29}$ Department of Epidemiology, German Institute of Human Nutrition, Potsdam, Germany; ${ }^{30}$ Institut National de la Sante et de la Recherche Médicale, Institut Gustave-Roussy, Villejuif, France; ${ }^{31}$ Institute of Community Medicine, University of Tromsǿ, Tromsǿ, Norway; and ${ }^{32}$ Department of Public Health and Clinical Medicine, Umeå University, Umeå, Sweden.

Address correspondence to Dr Gallo, Department of Epidemiology and Public Health, Imperial College London, St. Mary's Campus, Norfolk Place, W2 1PG, London, United Kingdom.

E-mail: v.gallo@imperial.ac.uk

Potential conflict of interest: Nothing to report. 
Amyotrophic lateral sclerosis (ALS) is a relentlessly progressive motor disease characterized by degeneration of the upper and lower motor neurons, leading to death in a median time of 3 years from disease onset, mainly because of complications of respiratory muscle weakness. ${ }^{1}$ ALS is sporadic in most patients, with reports of autosomal dominant inheritance in 5 to $10 \%$ of cases. About one quarter of familial cases are due to mutations of the copper-zinc superoxide dismutase (SOD1) gene. ${ }^{2}$ Causative SOD1 mutations are "gain of function," conferring toxicity to abnormal forms of the encoded protein. ${ }^{3}$

The incidence of ALS is of 1 to 3 per 100,000, and the prevalence is of 3 to 5 per 100,000 worldwide, ${ }^{1}$ with rare endemic foci reported in Guam, Papua New Guinea, and Kii peninsula in Japan. A major challenge in the study of causal association between putative risk factors and ALS is the rarity of the disease, which limits the use of cohort studies, the best study design for causal inference.

Cigarette smoking is the only risk factor that has been classified as "probable" in an evidence-based review of the literature of ALS risk factors. ${ }^{4}$ This was based on two class II studies" (ie, "cohort studies with parallel controls or case-control studies where bias or confounding may account for the findings about the risk factor in question, but not to an extent that would invalidate the findings completely" ${ }^{\prime 4}$ ), because data coming from large cohort studies were not available at the time. Subsequently, an increased risk for ALS death associated with smoking was shown in a case-control study conducted in Utrecht, the Netherlands ${ }^{7}$ and in women in a large cohort study conducted in the United States. ${ }^{8}$ Conversely, no association with either cigarette smoking or snuff dipping was found in a cohort of Swedish construction workers. ${ }^{9}$ Evidence on the role of cigarette smoking in ALS is still sparse and difficult to compare. ${ }^{5-9}$

Smoke from cigarettes contains a number of toxicants, many of which induce oxidative stress in the organism. Exhaled cigarette smoke has also been shown to contain formaldehyde, a substance generated from saccharides used as tobacco ingredients. ${ }^{10}$ A recent preliminary report has shown association of occupational exposure to formaldehyde with increased ALS mortality. ${ }^{11}$ Paraoxonases (PONs) are esterase enzymes with antioxidative properties that can be inhibited by cigarette smoking. ${ }^{12}$ Some polymorphisms associated with loss of function of PONs were found to be associated with ALS onset. ${ }^{13}$

The European Prospective Investigation into Cancer and Nutrition (EPIC) is a large cohort study, involving more than half a million subjects across 10 European countries, with a median follow-up time of 10 years. ${ }^{14}$ Originally designed for investigating cancer outcomes, EPIC is suitable for investigating other chronic diseases as well. At baseline, information on dietary habits and lifestyle risk factors (such as smoking patterns) were collected and are now available for analysis.

In this study, we investigated the following factors: (1) age-specific mortality rates from ALS in the EPIC cohort, and (2) the role of cigarette smoking on the risk for dying of ALS.

\section{Subjects and Methods \\ Population}

The 517,890 eligible healthy subjects were aged 35 to 70 years and recruited from the general population residing in a given geographical area in a period ranging from 1991 to 2001 , in 23 centers across 10 European countries ${ }^{14}$ as part of a large multicenter investigation on cancer and nutrition. Exceptions were the French cohort (based on women members of the health insurance for state school), the Ragusa, Sicily cohort (based on blood donors and their spouse), and most of the Oxford cohort (based on vegetarians). ${ }^{14}$ At recruitment, information on lifestyle and dietary habits was collected through standardized questionnaires. Follow-up for mortality and specific causes of death is conducted through linkage with death registries at regional and national levels; to date, the follow-up is virtually complete. ${ }^{14}$

All subjects were included in this analysis. Follow-up time was censored according to the proportion of reported causes of death: during each 6-month period, follow-up was censored when the cause of death was known for less than $80 \%$ of deaths, by center. This resulted in censoring follow-up time between December 2001 and December 2005, generating a total of 4,591,325 person-years.

\section{Exposure Assessment}

Data on smoking habits were collected at recruitment in the study, through a lifestyle questionnaire. All subjects were asked for the following information: (1) smoking status at recruitment (never, former, or current smoker) and (for most EPIC centers) number of cigarettes per day smoked at recruitment and at the ages of 20,30, 40, and 50 (ever smokers only); and (2) when they started smoking (ever smokers only), and when they quit smoking (former smokers only).

\section{Case Ascertainment}

ALS cases were defined as those subjects for whom "motor neuron disease" (G12.2 according to ICD-10 [International Classification of Diseases Tenth Revision] classification) was reported as an immediate, antecedent, or underlying cause of death.

\section{Statistical Analysis}

Mortality rates per 100,000 person-years in 5-year age bands were computed. For the follow-up time taken into consideration, an exit date was calculated as the date of death, of last contact, or of loss to follow-up. Single-subject follow-up time was calculated as the time elapsed between date of recruitment and exit date. For each age band, the contribution of years of follow-up of all participants entering the following 5-year follow-up period was added up. Number of ALS fatalities per age band was calculated, and age-specific mor- 
tality rates (per 100,000 person-years) were reported accordingly.

For the rest of the analysis, 12,411 subjects $(2.4 \%$ of the entire cohort) with missing information on smoking status at recruitment were excluded (including 2 ALS cases). Demographic characteristics of the entire cohort excluding ALS deaths and of the subjects who died of ALS were analyzed by sex; differences between all ALS cases and the rest of the cohort were tested with $\chi^{2}$ test for categorical variables and $t$ test for continuous variables. In particular, age at recruitment in the study, highest school title obtained, marital status, and employment were analyzed as potential confounders.

Smoking habits were analyzed for men and women separately among ALS cases and the rest of the cohort. Number of years spent smoking was calculated as age at recruitment (for current smokers) or age when quit smoking (for former smokers) minus age when started smoking. Similarly, time since quit smoking was calculated as age at recruitment minus the age when quit smoking for former smokers only. An estimation of a lifetime average number of cigarettes per day smoked was calculated as follows: for four age periods $(<30$, $30-39,40-49$, and $\geq 50$ years), the mean number of cigarettes smoked per day was calculated based on the questionnaire information. Similarly, for each period, the number of years spent smoking was calculated taking into account age at initiation, age at cessation, and age at recruitment. The lifetime mean number of cigarettes per day smoked was obtained by averaging the four periods. A cumulative lifetime smoking load was calculated for lifetime packs per years smoked (lifetime mean number of cigarettes smoked per day $X$ years spent smoking), defining a pack as containing 20 cigarettes. Proportion for categorical variables, means, and standard deviations for the continuous variables were calculated, and differences tested as described earlier.

Cox hazard models, with age as main time variable, were developed to investigate the role of smoking in the risk for dying of ALS. With the sample size available, the analysis had $85 \%$ power to detect as significant a hazard ratio $(\mathrm{HR}) \geq 1.4$ for risk factors having a $35 \%$ prevalence rate, or a $\mathrm{HR} \geq 1.6$ with a prevalence rate of $20 \%$, for a $5 \%$ type I error probability. The crude HR estimates were derived from a model in which the main exposure variable has been inserted together with sex, stratified by age and center of recruitment. The adjusted HR estimates derive from models in which other covariates (school level, marital status, employment status) were added in a stepwise fashion and the goodness of fit of each model compared with the previous through a likelihood ratio test (allowance being made for $p<0.05$ ), and stratified for age and center of recruitment. Given that cigarette smoking patterns among men and women changed greatly from the 40 s to the 90 s (with a progressive increase of women among smokers), all Cox models were repeated using calendar time as main time variable and were stratified by age at recruitment.

Duration of smoking for former and current smokers was divided in quartiles of distribution, and these compared using never smokers as reference. Crude and adjusted HR estimates were calculated accordingly; duration was also introduced as continuous variable in the model to calculate a $p$ value for trend. Similarly, time since quitting smoking in quartiles of distribution for former smokers only, taking as reference category the current smokers, was investigated in crude and adjusted Cox models. Number of packs per years smoked during lifetime was calculated for ever smokers and divided into quintiles of distribution. The quintiles were compared with never smokers as reference category, and a $p$ value for trend across categories was calculated. These definitions of smoking patterns were postulated a priori, and their classification was aimed at maximizing the power for the analysis.

\section{Results}

A total of 517,890 subjects, followed up for a median period of 10 years, were considered for the analysis, resulting in 4,591,325 person-years; during this period, a total of 118 ALS-related deaths were identified. For 110 subjects (93.2\%), ALS was coded as the underlying cause of death, for 7 subjects $(5.9 \%)$ as the immediate (or direct) cause of death, and for 1 subject $(0.8 \%)$ as the antecedent cause of death. Furthermore, ALS was recorded as "other cause of death" in four subjects who died of cardiac surgery (ICD-10: Y-831), a cerebrovascular event (I-64), chronic obstructive pulmonary disease (J-449), and Hodgkin's lymphoma (C859); and it was found to be recorded in death records (without specification of the role) in four additional cases of death caused by pulmonary embolism after lower limbs phlebitis (1-269, I-803), viral infection (B$338)$, dementia (F-03), and sequelae of poliomyelitis (B-91). These last eight subjects were not included in the analysis because ALS diagnosis was considered to be unreliable.

Age-specific mortality rates per 100,000 subjects were calculated among the entire EPIC population, resulting in a crude mortality rate of 2.69 per 100,000 person-years. Figure 1 shows age- and sex-specific crude mortality rates of ALS, with a steep increase in

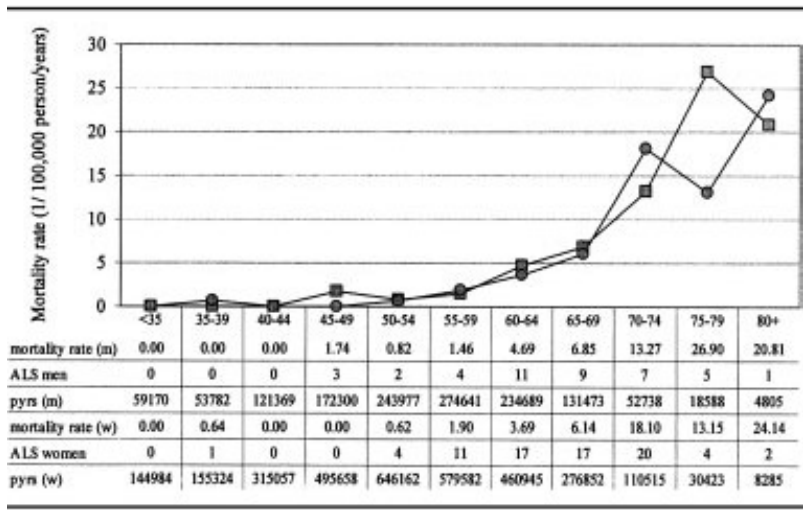

Fig 1. Age-specific mortality rates (in 5-year age bands, per 100,000 person-years) of amyotrophic lateral sclerosis (ALS) in the European Prospective Investigation into Cancer and Nutrition (EPIC) population for men (squares) and women (circles). The table lists mortality rate, number of ALS cases, and person-years (pyrs) per each 5-year age band for men $(m)$ and women $(w)$. 
the incidence with increasing age, in particular, after the age of 70, and a subsequent decline in the eldest ( $\geq 85$ years old). However, the reduced number of person-years available in this latter group does not allow a solid estimation of the age-specific incidence rate. Age-adjusted ALS incidence rate obtained after direct standardization on the World Standard Population truncated to 35 to 75 age groups is 2.28 per 100,000 person-years.

In the analysis of smoking habits, a total of 505,479 subjects $(97.6 \%$ of the original cohort, comprising 116 ALS cases) were included (for a total 4,492,031 person-years), allowing for missing data on smoking habits at recruitment. The models including smoking duration included 492,630 subjects $(97.5 \%$ of the sample), and the model including the amount of cigarettes smoked included 431,984 subjects $(85.5 \%$ of the sample) allowing for missing values. Overall, the proportion of missing data is low and unlikely to substantially bias the results. Subjects who died of ALS during the follow-up period were, on average, 10 years older then the rest of the cohort at recruitment $(59.8$ vs 51.0 years; $p<0.001$ ) (Table 1). ALS cases were more likely to have no or primary education when compared with the rest of the cohort $(p=0.007)$ (see Table 1); also, there was a greater proportion of widows among the ALS cases, but these differences could be partly explained by the older age at recruitment (see Table 1). Finally, only $41 \%$ of ALS cases were employed at recruitment compared with $67 \%$ of the rest of the cohort $(p<0.001)$ (see Table 1$)$.

At recruitment, only 49 (42\%) ALS cases declared to be never smokers compared with $50 \%$ of the rest of the cohort; this difference was larger among men: $23 \%$ of men who died of ALS were never smokers compared with $33 \%$ in the rest of the cohort (see Table 1). ALS cases spent, on average, 7 more years smoking than the rest of the cohort ( 31.2 vs 24.0 years; $p<0.001$ ). This difference was significant both in men (33.2 vs 26.5 years; $p=0.003$ ) and in women (29.9 vs 22.4 years; $p=0.005)$. There was no significant difference between the mean number of cigarettes per day smoked at recruitment between ALS cases and the rest of the cohort (12.6 vs 14.7 cigarettes/day; $p=0.495$ ), or significant difference between the estimated lifetime mean number of cigarettes per day smoked by ALS cases (13.6; standard deviation, 8.9) and by the rest of the cohort (13.3; standard deviation, 8.4) $(p=0.805)$. Time since quitting smoking was not significantly different between ALS cases and the rest of the cohort among former smokers $(p=0.113)$ (see Table 1 ).

The proportional hazard assumption was verified and holds true. Smoking status, as recorded at recruitment, was significantly associated with the risk for dying of ALS during follow-up: current smokers at recruitment in the study had an almost twofold increased risk for dying of ALS compared with never smokers (HR, 1.89; 95\% confidence interval [CI], 1.14-3.14; $p=0.014)$, whereas former smokers had a roughly $50 \%$ increased risk (HR, 1.48; 95\% CI, 0.94-3.32; $p=0.089$ ) compared with never smokers (Table 2). When analyzed separately, the direction of the association was preserved in both men and women, although running short of statistical significance caused by the reduction in power. The number of years spent smoking increased the risk for dying of ALS ( $p$ for trend $=$ $0.002)$ in both crude and adjusted models. Those who smoked more than 33 years had a more than twofold increased risk for dying of ALS compared with never smokers (HR, 2.16; 95\% CI, 1.32-3.54; $p=0.002$ ). This association was confirmed when considering men ( $p$ for trend $=0.059$ ) and women ( $p$ for trend $=$ 0.015 ) separately despite the reduction in power (see Table 3 online and Fig 2). Also, this estimate did not change substantially when the estimated lifetime mean number of cigarettes per day smoked was added in the model, suggesting a stronger effect of smoking duration than of dose in increasing the risk for ALS. This is also confirmed by the fact that a measure of lifetime smoking load (which takes into consideration both duration and amount of smoking) runs short of statistical significance in relation to ALS mortality ( $p$ for trend across quintiles $=0.118$ ), although it is significant among women ( $p$ for trend $=0.046$ ) (see Table 3 online). Conversely, the number of years since quitting smoking, in quartiles of distribution, was associated with decreased risk for dying of ALS compared with those who were still smoking at the moment of recruitment. Despite small numbers, a borderline significant trend of risk reduction with an increasing number of years since quitting smoking was evident in men ( $p$ for trend $=0.067$ ) but not in women, resulting in a nonsignificant association in the entire cohort. However, a trend toward a reduced risk with an increasing number of years since quitting smoking is suggested ( $p$ for trend $=0.125$ ), which would be coherent with a model of risk reduction after the exposure cessation (see Table 2 and Fig 2). Cox models analyses using calendar time as main time variable did not change estimates and level of significance (result not shown).

A sensitivity analysis conducted after including those eight subjects for whom ALS was reported among "other" or "unspecified causes of death" showed increased power. The $p$ value for linear trend with time since quitting smoking is of borderline statistical significance $(p=0.005)$, as well as the $p$ value for packyears $(p=0.032)$

\section{Discussion}

These results suggest that smoking is likely to be a risk factor for ALS. The clear and significant dose-response relation between number of years spent smoking and 
Table 1. Demographic Characteristics and Smoking Habits of the Sample

\begin{tabular}{|c|c|c|c|c|c|c|c|}
\hline \multirow[t]{2}{*}{ Characteristics } & \multicolumn{2}{|c|}{$\operatorname{ALS}(\mathbf{N}=116)$} & \multicolumn{2}{|c|}{ EPIC Cohort ${ }^{a}(\mathrm{~N}=505,355)$} & \multirow{2}{*}{$\begin{array}{c}\text { ALS } \\
\text { All } \\
(N=116)\end{array}$} & \multirow{2}{*}{$\begin{array}{c}\text { EPIC Cohort }{ }^{\mathrm{a}} \\
\text { All } \\
(\mathrm{N}=505,355)\end{array}$} & \multirow[t]{2}{*}{$p^{b}$} \\
\hline & $\begin{array}{c}\text { Male } \\
\text { Subjects } \\
(\mathrm{n}=40)\end{array}$ & $\begin{array}{l}\text { Female } \\
\text { Subjects } \\
(n=76)\end{array}$ & $\begin{array}{c}\text { Male } \\
\text { Subjects } \\
(\mathbf{n}=149,277)\end{array}$ & $\begin{array}{c}\text { Female } \\
\text { Subjects } \\
(\mathbf{n}=356,078)\end{array}$ & & & \\
\hline $\begin{array}{l}\text { Mean age at } \\
\text { recruitment }(\mathrm{SD}), \mathrm{yr}\end{array}$ & $59.2(8.1)$ & $60.1(8.2)$ & $52.0(10.2)$ & $50.6(9.9)$ & $59.8(8.1)$ & $51.0(10.0)$ & $<0.001$ \\
\hline \multicolumn{8}{|l|}{$\begin{array}{l}\text { Smoking status at } \\
\text { recruitment, } \mathrm{n}(\%)\end{array}$} \\
\hline Never smoker & $9(22.5)$ & $40(52.6)$ & $49,522(33.2)$ & $201,695(56.6)$ & $49(42.2)$ & $251,217(49.7)$ & 0.181 \\
\hline Former smoker & $17(42.5)$ & $23(30.3)$ & $55,508(37.2)$ & $83,027(23.3)$ & $40(34.5)$ & $138,535(27.4)$ & \\
\hline Current smoker & $14(35.0)$ & $13(17.1)$ & $44,247(29.6)$ & $71,356(20.4)$ & $27(23.3)$ & $115,603(22.9)$ & \\
\hline $\begin{array}{l}\text { Mean years spent } \\
\text { smoking }(\mathrm{SD})^{\mathrm{c}, \mathrm{d}}\end{array}$ & $33.2(15.0)$ & $29.4(12.6)$ & $26.5(12.5)$ & $22.4(11.8)$ & $31.2(13.8)$ & $24.0(12.2)$ & $<0.001$ \\
\hline $\begin{array}{l}\text { Mean cigarettes/day } \\
\text { at recruitment }(S D)^{c, c}\end{array}$ & $13.0(8.8)$ & $13.8(7.6)$ & $17.4(10.9)$ & $13.3(7.9)$ & $12.6(7.9)$ & $14.7(9.3)$ & 0.495 \\
\hline $\begin{array}{l}\text { Mean cigarettes/day } \\
\text { lifetime }(\mathrm{SD})^{\mathrm{c}, \mathrm{f}}\end{array}$ & $15.6(9.5)$ & $12.0(8.3)$ & $16.3(9.4)$ & $11.2(6.9)$ & $13.6(8.9)$ & $13.3(8.4)$ & 0.805 \\
\hline $\begin{array}{l}\text { Time since quit } \\
\text { smoking (SD), } \mathrm{yr}^{\mathrm{g}}\end{array}$ & $15.9(15.7)$ & $19.4(10.2)$ & $15.7(10.7)$ & $15.0(10.1)$ & $17.9(12.8)$ & $15.3(10.3)$ & 0.113 \\
\hline \multicolumn{8}{|l|}{ School level, n (\%) } \\
\hline None/Primary & $21(52.5)$ & $31(40.8)$ & $50,807(34.0)$ & $102,512(28.8)$ & $52(44.5)$ & $153,319(30.3)$ & 0.007 \\
\hline Technical & $7(17.5)$ & $13(17.1)$ & $36,475(24.4)$ & $77,342(21.7)$ & $20(17.2)$ & $113,817(22.5)$ & \\
\hline Secondary & $3(7.5)$ & $17(22.4)$ & $19,293(12.9)$ & $82,389(23.1)$ & $20(17.2)$ & $101,682(20.1)$ & \\
\hline University & $8(20.0)$ & $10(13.2)$ & $39,132(26.2)$ & $80,130(22.5)$ & $18(15.5)$ & $119,261(23.6)$ & \\
\hline Unknown & $1(2.5)$ & $5(6.6)$ & $3,571(2.4)$ & $13,705(3.9)$ & $6(5.2)$ & $17,276(3.4)$ & \\
\hline \multicolumn{8}{|l|}{ Marital status, $\mathrm{n}(\%)^{\mathrm{h}}$} \\
\hline Single & $0(0.0)$ & $7(11.1)$ & $11,327(10.8)$ & $32,190(11.1)$ & $7(7.4)$ & $43,517(11.0)$ & 0.030 \\
\hline Married & $25(78.1)$ & $47(74.6)$ & $84,559(80.8)$ & $226,835(78.0)$ & $80(75.8)$ & $311,394(78.7)$ & \\
\hline Separated/divorced & $4(12.5)$ & $3(4.8)$ & $6,934(6.6)$ & $18,349(6.3)$ & $7(7.4)$ & $25,283(6.4)$ & \\
\hline Widowed & $3(9.4)$ & $6(9.5)$ & $1,878(1.8)$ & $13,538(4.7)$ & $9(9.5)$ & $15,416(3.9)$ & \\
\hline \multicolumn{8}{|l|}{ Employment, $\mathrm{n}(\%)^{\mathrm{i}}$} \\
\hline $\begin{array}{l}\text { Employed at } \\
\text { recruitment }\end{array}$ & $20(58.8)$ & $19(30.7)$ & $97,341(73.7)$ & $207,862(64.3)$ & $39(40.6)$ & $305,203(67.0)$ & $<0.001$ \\
\hline \multicolumn{8}{|c|}{ Excluding amyotrophic lateral sclerosis (ALS) cases. } \\
\hline \multicolumn{8}{|c|}{$\begin{array}{l}\mathrm{b} p \text { value for } \chi^{2} \text { test for discrete variables, and for } t \text { test against the } \mathrm{H}_{0} \text { : difference of means }=0 \text { for continuous variables, in both cases } \\
\text { comparing subjects who died of ALS with the rest of the cohort. } \\
\text { cFor ever smokers only }(\mathrm{n}=254,213) \text {. }\end{array}$} \\
\hline \multicolumn{8}{|c|}{$\begin{array}{l}\text { d Data missing for } 12,849(5.1 \%) \text { subjects. } \\
\text { eData missing for } 10,350(9.0 \%) \text { subjects. }\end{array}$} \\
\hline \multicolumn{8}{|c|}{$\begin{array}{l}{ }^{f} \text { Data missing for } 85,905(16.6 \%) \text { subjects. } \\
\text { 'For former smokers only }(n=138,580) \text {, data missing for } 5,774(4.2 \%) \text { subjects. }\end{array}$} \\
\hline \multicolumn{8}{|c|}{$\begin{array}{l}\text { h} \text { Missing values for } 109,766 \text { subjects }(21.7 \%) \text {. } \\
\text { i Missing values for } 49,802 \text { subjects }(9.9 \%) \text {. }\end{array}$} \\
\hline $\mathrm{IC}=$ European Pro & rive Investi & ion into $\mathrm{C}$ & r and Nutritio & D $=$ stanc & & & \\
\hline
\end{tabular}

the risk for ALS contributes to strengthen the inference. This is further supported by a statistically nonsignificant but inverse trend with increasing years since quitting smoking; the lack of significance might be, at least partly, caused by reduced power after exclusion of never smokers from this model. Conversely, the amount of cigarette smoke exposure appears to play a less important role on the risk for ALS when compared with length of exposure. Similar patterns have been de- scribed for the effect of smoking on other chronic diseases such as lung cancer: The number of years spent smoking is, in fact, more predictive than any other smoking pattern on development of lung cancer (including lifetime number of cigarettes smoked). ${ }^{15,16}$

The time trends observed in our study, that is, an increased risk with increasing years spent smoking and a decreased risk with increasing years after quitting smoking, are suggestive of a causal role of smoking in 


\begin{tabular}{|c|c|c|c|c|c|c|c|c|}
\hline Characteristics & $\begin{array}{c}\text { ALS } \\
\text { Cases }\end{array}$ & $\begin{array}{l}\text { Person- } \\
\text { Years }\end{array}$ & $\begin{array}{l}\text { Crude } \\
\text { HR }^{2}\end{array}$ & $95 \% \mathrm{CI}$ & $p$ & $\begin{array}{c}\text { Adjusted } \\
\text { HR }^{\mathbf{b}}\end{array}$ & $95 \% \mathrm{CI}$ & $p$ \\
\hline \multicolumn{9}{|c|}{ Smoking status at recruitment ${ }^{c}$} \\
\hline Never smokers & 49 & $2,270,062$ & Reference & - & - & Reference & - & - \\
\hline Former smokers & 40 & $1,217,080$ & 1.43 & $0.92-2.24$ & 0.114 & 1.48 & $0.94-2.32$ & 0.089 \\
\hline Current smokers & 27 & $1,004,850$ & 1.89 & $1.14-3.13$ & 0.013 & 1.89 & $1.14-3.14$ & 0.014 \\
\hline \multicolumn{9}{|l|}{ Time spent smoking ${ }^{d}$} \\
\hline Never smokers & 49 & $2,270,062$ & Reference & - & - & Reference & - & - \\
\hline$<14$ yr & 8 & 482,267 & 1.16 & $0.54-2.50$ & 0.695 & 1.21 & $0.56-2.61$ & 0.622 \\
\hline $14-23 \mathrm{yr}$ & 10 & 533,265 & 1.27 & $0.63-2.56$ & 0.504 & 1.32 & $0.65-2.66$ & 0.438 \\
\hline $24-32 \mathrm{yr}$ & 13 & 527,415 & 1.55 & $0.82-2.93$ & 0.177 & 1.59 & $0.84-3.01$ & 0.155 \\
\hline$\geq 33 \mathrm{yr}$ & 33 & 562,295 & 2.16 & $1.33-3.53$ & 0.002 & 2.16 & $1.32-3.54$ & 0.002 \\
\hline Trend & & & & & 0.002 & & & 0.002 \\
\hline \multicolumn{9}{|l|}{ Time since quit smoking ${ }^{\mathrm{e}}$} \\
\hline Current smokers & 27 & $1,004,850$ & Reference & - & - & Reference & - & - \\
\hline$<6 \mathrm{yr}$ & 8 & 278,803 & 0.93 & $0.42-2.06$ & 0.856 & 0.94 & $0.43-2.09$ & 0.888 \\
\hline $6-13 \mathrm{yr}$ & 10 & 290,228 & 0.93 & $0.44-1.95$ & 0.852 & 0.94 & $0.45-1.97$ & 0.865 \\
\hline $14-22 \mathrm{yr}$ & 9 & 305,832 & 0.63 & $0.29-1.38$ & 0.251 & 0.64 & $0.29-1.41$ & 0.273 \\
\hline$>22 \mathrm{yr}$ & 12 & 291,937 & 0.58 & $0.28-1.19$ & 0.137 & 0.60 & $0.29-1.24$ & 0.167 \\
\hline Trend & & & & & 0.101 & & & 0.125 \\
\hline \multicolumn{9}{|l|}{ Packs/years smoked $^{\mathrm{f}}$} \\
\hline Never smokers & 49 & $2,270,062$ & Reference & - & - & Reference & - & - \\
\hline$<5$ packs $/$ yr & 5 & 305,098 & 1.06 & $0.41-2.76$ & 0.898 & 1.13 & $0.43-2.93$ & 0.804 \\
\hline 5-10 packs/yr & 8 & 311,767 & 1.65 & $0.74-3.66$ & 0.218 & 1.70 & $0.76-3.76$ & 0.194 \\
\hline 11-18 packs/yr & 5 & 296,881 & 1.19 & $0.45-3.12$ & 0.724 & 1.22 & $0.46-3.20$ & 0.689 \\
\hline 19-27 pack/yr & 10 & 312,492 & 1.88 & $0.89-3.94$ & 0.097 & 1.87 & $0.89-3.94$ & 0.099 \\
\hline \multirow[t]{2}{*}{$\geq 28$ packs $/ \mathrm{yr}$} & 12 & 314,902 & 1.54 & $0.74-3.24$ & 0.251 & 1.51 & $0.72-3.19$ & 0.279 \\
\hline & & & & & 0.104 & & & 0.118 \\
\hline \multicolumn{9}{|c|}{ 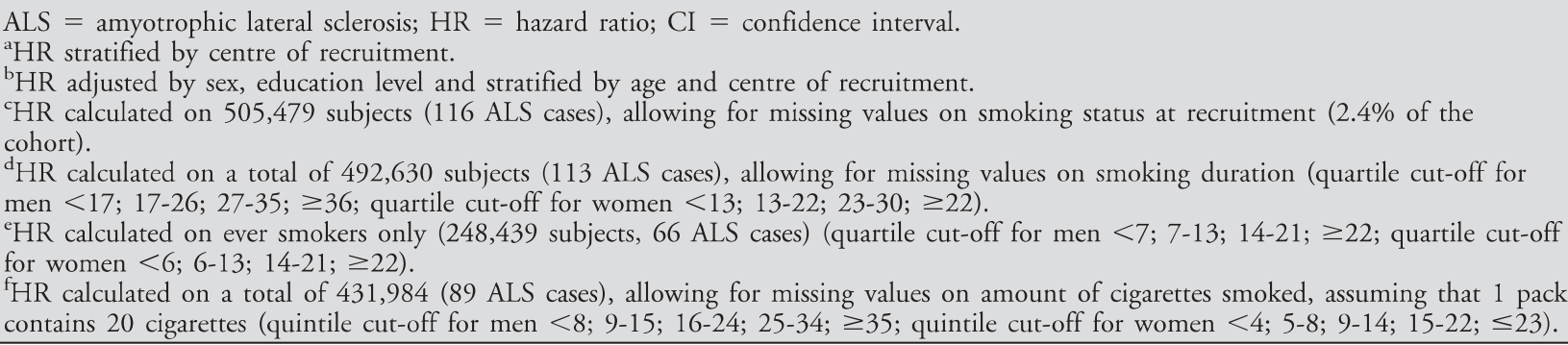 } \\
\hline
\end{tabular}

ALS. A probable reason why the association with time since quitting smoking does not reach statistical significance is the relatively small number of subjects in the former smoker category. This category may also potentially suffer from some degree of misclassification, because some subjects classified as current smokers at recruitment (thus included in the reference category) could have quit during the follow-up period. At odds with the results coming from the Cancer Prevention study, ${ }^{7}$ our data suggest that the effect of smoking on the risk for dying of ALS is the same in both sexes, because the interaction term with sex was not significant, and the estimates were consistent between men and women. School level was strongly associated with 


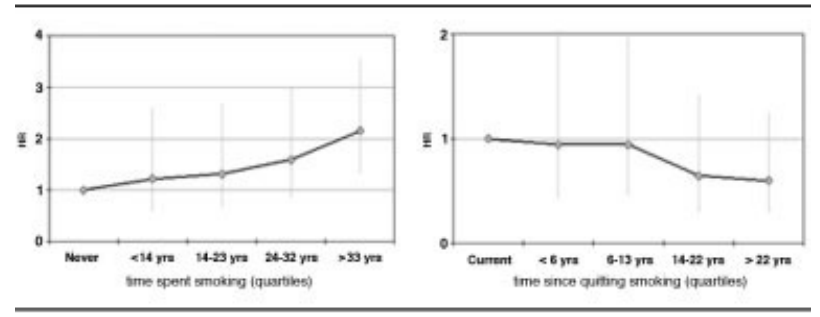

Fig 2. Adjusted hazard ratio (HR) and relative 95\% confidence interval of dying of amyotrophic lateral sclerosis (ALS) according to the number of years spent smoking (using never smokers as reference) (left) and the number of years since quitting smoking (using current smokers at recruitment as reference) (right).

the risk for dying of ALS even after adjusting for both smoking status at recruitment and duration of smoking (results not shown). This observation, which was also reported by others, ${ }^{8,17}$ might represent the effect of some residual confounding from smoking and/or another environmental factor, distributed unevenly across social strata, which might modify the individual risk for development of the disease. However, the small changes in the estimates of the HR of dying of ALS being a smoker once adjusted by education (shown in Table 2) suggests only a small confounding effect of education on the association between smoking and ALS. These figures contribute to orientate the research toward the study of other environmental risk factors for ALS in addition to cigarette smoking.

Age-specific incidence rates of ALS in this study are comparable with those found in other European studies $^{9,18,19}$ except for a delayed decrease in incidence, after the age of 79 rather than after age 75 , as was observed previously. This might be because of the fact that the mortality rates were plotted instead of the incidence rates. Although, in absolute terms, mortality is a good approximation for incidence; given the almost invariable $100 \%$ fatality rate of the disease, the median disease duration of about 3 years can shift the age-specific rates calculated over 5-year age bands. Also, if younger subjects tend to have a longer survival time, their mortality will shift the mortality peak toward older ages.

Main limitations of this study are the small sample size of subjects who died of ALS, the assessment of exposures up to the time of recruitment, and the assessment of the outcome through death certificates only. Exposure assessment performed at recruitment only would not allow recording changes in the exposure status, thus biasing any association toward the null. Death certificates have been shown to be generally accurate regarding the diagnosis of ALS: previous studies conducted in the United States ${ }^{7}$ confirmed that virtually all deaths coded with 335.2 (motor neuron disease, according to ICD-9) and G12.2 (motor neuron disease, according to ICD-10) were actually diagnosed as ALS
(98.3\%) or bulbar palsy (1.1\%). Similarly, another study conducted in Europe on the validity of hospital discharge diagnosis for ALS reported a sensitivity of $91.6 \%$ and a specificity of $99.9 \%$ for the ICD- 9 code. ${ }^{20}$ Death certificates were also found to be adequate in describing ALS mortality in a study in Italy. ${ }^{2}$

At least four recognizable factors have contributed to improved ascertainment of ALS over the past 20 years: (1) increased public awareness of the disease, (2) increased awareness by the aging population that progressive weakness is not a normal part of aging, (3) increased number of neurologists; and (4) increased availability of electrodiagnostic and radiological diagnostic techniques. ${ }^{1}$ Referral bias contribution to case ascertainment is estimated to be low: ALS is a progressive disabling neurological disease that warrants typically specialized evaluation at some stage of disease progression; general practitioners and other physicians tend to refer patients with such relentlessly paralytic conditions to specialists; for neurologists, the diagnosis of ALS in its advanced stage is usually straightforward. Also, residual confounding from unmeasured factors cannot be completely ruled out, in particular that caused by occupational exposure to toxicants. Although the sample size is limited, the prospective collection of data adds value to our results, which are free from recall bias.

Formaldehyde exposure was recently proposed as significantly associated with ALS, with a dose-response relation with increasing years of exposure ${ }^{11}$; these results appear consistent with these findings. It was previously observed that pyramidal cells are vulnerable to formaldehyde exposure in newborn rats, ${ }^{22,23}$ and that aldehydes may potentially play a role in $\beta$-amyloid aggregation related to Alzheimer's disease pathology. ${ }^{24}$ Exposure to formaldehyde induces oxidative stress mainly via lipid peroxidation in the brain. ${ }^{25,26}$ Lipid peroxidation has also recently been implicated in ALS causative mechanisms: the PONs PON1, PON2, and PON3 are esterase enzymes with antioxidant properties preventing low-density lipoprotein from peroxidation; some polymorphisms with a loss of function of PONs were described as associated with ALS. ${ }^{13,27}$ Also, the activity of PONs is inhibited by cigarette smoking. ${ }^{12,28}$

In conclusion, the consistency of our observations strongly supports the hypothesis of cigarette smoking in the cause of ALS. This effect might be exerted through oxidative stress and lipid peroxidation (perhaps, at least partly, induced by formaldehyde exposure). This is also strongly supported by the significant linear dose-response relation of risk for ALS with years of exposure to tobacco smoke.

This research has been made possible thanks to a grant of the European Community (5th Framework Programme) to Prof. Paolo Vineis (grant QLK4CT199900927); and a grant of the Compagnia di San Paolo to the ISI Foundation (PV, AM, DP, SS, RT.) 
All authors are independent form founders. Mortality data from the Netherlands are obtained from "Statistics Netherlands".

In addition we would like to thank for their financial support:

Europe Against cancer Program of the European Commission (SANCO); ISCIII, Red de Centros RCESP, C03/09 (JMJM, LA, ABG); Deutsche Krebshilfe (JL, RK); Deutsches Krebsforschungszentrum (JL, RK, MMB, HB); German Federal Ministry of Education and Research (JL, RK, MMB, HB); Danish Cancer Society (AT, AO, KO); Health Research Fund (FIS) of the Spanish Ministry of Health (JMJM, MJTD, LRS, AA, LA, ABG); Spanish Regional Governments of Andalucia, Asturias, Basque Country, Murcia and Navarra (JMJM, MJTD, LRS, LA, ABG); Red Temática de Investigación Cooperativa en Cáncer (RTICC), ISCIII, Spanish Ministry of Science and Innovation (grant RD06/0020/0091) (AA); Cancer Research U.K. (NEA, AWR, SB, KTK); Medical Research Council, United Kingdom (B, KTK, ER, PV, LM, VG); Stroke Association, United Kingdom (SB, KTK, ER, PV, LM, VG).

British Heart Foundation (SB, KTK, ER, PV, LM, VG); Department of Health, United Kingdom (SB, KTK, ER, PV, LM, VG); Food Standards Agency, United Kingdom (SB, KTK, ER, PV, LM, VG); Wellcome Trust, United Kingdom (SB, KTK, ER, PV, LM, VG); Greek Ministry of Health (AK, AT); Greek Ministry of Education (AK, AT); Italian Association for Research on Cancer (AIRC); Italian National Research Council (PV, AM, DP, SS, RT); Dutch Ministry of Public Health, Welfare and Sports (HBBDM, HCB); World Cancer Research Fund; Swedish Cancer Society (JM, BJ, GH, PA); Swedish Scientific Council (JM, BJ, GH, PA); Regional Government of Skåne and Västerbotten, Sweden (JM, BJ, GH, PA); Norwegian Cancer Society (EL); Research Council of Norway (EL); French League against cancer, Inserm, Mutuelle Generale l'Education National and IGR (FCC).

\section{References}

1. Armon C. Amyotrophic lateral sclerosis. In: Nelson LM, Tanner CM, Van Den Eeden SK, et al, eds. Neuroepidemiology. From principles to practice. Oxford: Oxford University Press, 2004:162-187.

2. Rosen DR, Sapp P, O’Regan J, et al. Genetic linkage analysis of familial amyotrophic lateral sclerosis using human chromosome 21 microsatellite DNA markers. Am J Med Genet 1994;51: 61-69.

3. Valentine JS, Hart PJ. Misfolded CuZnSOD and amyotrophic lateral sclerosis. Proc Natl Acad Sci U S A 2003;100: 3617-3622.

4. Armon C. An evidence-based medicine approach to the evaluation of the role of exogenous risk factors in sporadic amyotrophic lateral sclerosis. Neuroepidemiology 2003;22:217-228.

5. Kamel F, Umbach DM, Munsat TL, et al. Association of cigarette smoking with amyotrophic lateral sclerosis. Neuroepidemiology 1999;18:194-202.

6. Nelson LM, McGuire V, Longstreth WT Jr, et al. Populationbased case-control study of amyotrophic lateral sclerosis in western Washington State. I. Cigarette smoking and alcohol consumption. Am J Epidemiol 2000;151:156-163.

7. Sutedja NA, Veldink JH, Fischer K, et al. Lifetime occupation, education, smoking, and risk of ALS. Neurology 2007;69: $1508-1514$

8. Weisskopf MG, McCullough ML, Calle EE, et al. Prospective study of cigarette smoking and amyotrophic lateral sclerosis. Am J Epidemiol 2004;160:26-33.

9. Fang F, Bellocco R, Hernan MA, et al. Smoking, snuff dipping and the risk of amyotrophic lateral sclerosis-a prospective cohort study. Neuroepidemiology 2006;27:217-221.
10. Baker RR. The generation of formaldehyde in cigarettes - overview and recent experiments. Food Chem Toxicol 2006;44: 1799-1822.

11. Weisskopf M, Morozova N, O'Reilly E, et al. Prospective study of chemical exposures and amyotrophic lateral sclerosis mortality (Abstract). American Academy of Neurology 60th Annual Meeting; Chicago, Illinois, 2008.

12. Nishio E, Watanabe Y. Cigarette smoke extract inhibits plasma paraoxonase activity by modification of the enzyme's free thiols. Biochem Biophys Res Commun 1997;236:289-293.

13. Slowik A, Tomik B, Wolkow PP, et al. Paraoxonase gene polymorphisms and sporadic ALS. Neurology 2006;67:766-770.

14. Riboli E, Hunt KJ, Slimani N, et al. European Prospective Investigation into Cancer and Nutrition (EPIC): study populations and data collection. Public Health Nutr 2002;5: 1113-1124.

15. Doll R, Peto R. Cigarette smoking and bronchial carcinoma: dose and time relationships among regular smokers and lifelong non-smokers. J Epidemiol Community Health 1978;32: 303-313.

16. Flanders WD, Lally CA, Zhu BP, et al. Lung cancer mortality in relation to age, duration of smoking, and daily cigarette consumption: results from Cancer Prevention Study II. Cancer Res 2003;63:6556-6562.

17. Longstreth WT, McGuire V, Koepsell TD, et al. Risk of amyotrophic lateral sclerosis and history of physical activity: a population-based case-control study. Arch Neurol 1998;55: 201-206.

18. Logroscino G, Traynor BJ, Hardiman O, et al. Descriptive epidemiology of amyotrophic lateral sclerosis: new evidence and unsolved issues. J Neurol Neurosurg Psychiatry 2008;79:6-11.

19. Traynor BJ, Codd MB, Corr B, et al. Incidence and prevalence of ALS in Ireland, 1995-1997: a population-based study. Neurology 1999;52:504-509.

20. Beghi E, Logroscino G, Micheli A, et al. Validity of hospital discharge diagnoses for the assessment of the prevalence and incidence of amyotrophic lateral sclerosis. Amyotroph Lateral Scler Other Motor Neuron Disord 2001;2:99-104.

21. Chio A, Magnani C, Oddenino E, et al. Accuracy of death certificate diagnosis of amyotrophic lateral sclerosis. J Epidemiol Community Health 1992;46:517-518.

22. Gurel A, Coskun O, Armutcu F, et al. Vitamin E against oxidative damage caused by formaldehyde in frontal cortex and hippocampus: biochemical and histological studies. J Chem Neuroanat 2005;29:173-178.

23. Sarsilmaz M, Kaplan S, Songur A, et al. Effects of postnatal formaldehyde exposure on pyramidal cell number, volume of cell layer in hippocampus and hemisphere in the rat: a stereological study. Brain Res 2007;1145:157-167.

24. Chen K, Maley J, Yu PH. Potential implications of endogenous aldehydes in beta-amyloid misfolding, oligomerization and fibrillogenesis. J Neurochem 2006;99:1413-1424.

25. Maboudou P, Mathieu D, Bachelet H, et al. Detection of oxidative stress. Interest of GC-MS for malondialdehyde and formaldehyde monitoring. Biomed Chromatogr 2002;16: 199-202.

26. Zararsiz I, Kus I, Akpolat N, et al. Protective effects of omega-3 essential fatty acids against formaldehyde-induced neuronal damage in prefrontal cortex of rats. Cell Biochem Funct 2006; 24:237-244.

27. Saeed M, Siddique N, Hung WY, et al. Paraoxonase cluster polymorphisms are associated with sporadic ALS. Neurology 2006;67:771-776.

28. Costa LG, Vitalone A, Cole TB, et al. Modulation of paraoxonase (PON1) activity. Biochem Pharmacol 2005;69:541-550. 